\title{
Burkitt lymphoma - Nutritional support during induction treatment: Effect on anthropometric parameters and morbidity of treatment
}

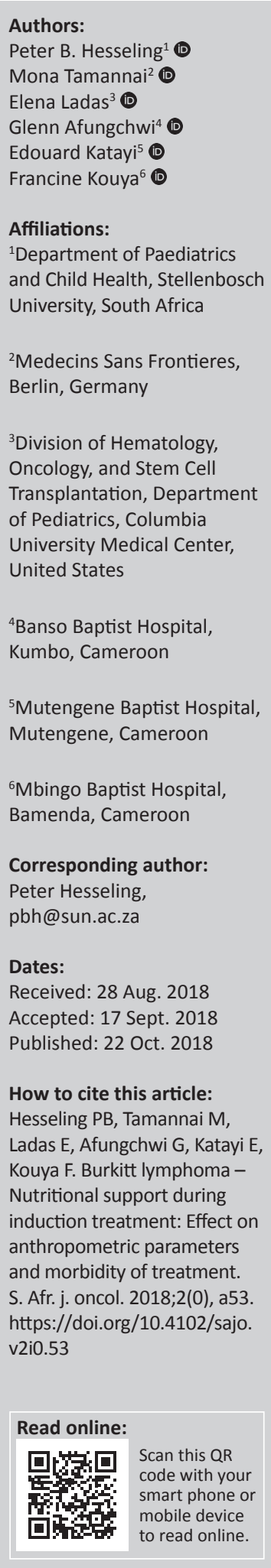

Background: Malnutrition is common in children diagnosed with cancer in Africa, and it adds to the morbidity and mortality of treatment. Nutritional support is known to reduce morbidity and mortality of treatment.

Aim: The aim of this study was to record changes in anthropometric parameters, morbidity and mortality in patients admitted with Burkitt lymphoma (BL) whose diet was supplemented with protein, vitamins and minerals during induction chemotherapy.

Methods: Seventy consecutive newly diagnosed BL patients were enrolled. The diet was supplemented with a daily egg, $200 \mathrm{~mL}$ F-75 formula and vitamins. Guardians received 3 cups of dry rice and $\$ 1$ daily to buy and prepare meals for the patient and themselves whilst in the hospital. Height, weight, triceps skinfold (TSF) and mid-upper arm circumference (MUAC) were recorded on days 1 and 28. Co-morbidities at diagnosis were treated, and neutropenia and febrile episodes managed with a standard protocol. Two patients who died within $24 \mathrm{~h}$ after admission were excluded from the anthropometric analyses.

Results: The mean age was 8 (range 2-16) years and the male:female ratio was $42: 28$. The St Jude stage distribution was as follows: Stage I $=6 \%$, II $=4 \%, \mathrm{III}=69 \%, \mathrm{IV}=21 \%$. Weight for age was $<10$ th centile at diagnosis in $18 \%$ (but influenced by tumour mass). Weight was unchanged or increased by $\geq 5 \%$ in $66 \%$ of patients on Day 28 . The TSF was $<3$ rd centile in $47 \%$ of patients and increased by $\geq 0.5 \mathrm{~cm}$ in $57 \%$. The MUAC was $<3$ rd centile in $16 \%$ of patients at diagnosis and in $10 \%$ of patients on Day 28 . Febrile episodes in $60 \%$ and neutropenia in $18 \%$ of patients were successfully treated. Two patients died from presumed renal failure. The overall death rate (including the two deaths within $24 \mathrm{~h}$ after admission before chemotherapy was started) was $5.5 \%(n=4)$.

Conclusion: The TSF improved in the majority and the MUAC improved in some patients. Febrile neutropenia and febrile episodes could be successfully managed. The death rate during induction was lower than in our previously published results with the same chemotherapy protocol. Dietary supplementation should be a standard component of treatment in paediatric patients with cancer.

\section{Introduction}

Endemic Burkitt lymphoma (BL), a highly aggressive lymphoma, is the most common childhood cancer in sub-Saharan Africa. The age standardised rate is 3.3 in the sub-Saharan region and 2.6 in the north-west region of Cameroon. ${ }^{1,2}$

Limited resources, such as unaffordable or unavailable drugs and limited supportive care, have led to the development of treatment with high frequency cyclophosphamide (CPM) during induction therapy. A $61 \%$ one-year disease-free survival rate was recorded in Cameroon with this treatment protocol, compared to survival rates of $\geq 80 \%$ in developed countries. ${ }^{3,4,5}$

Families in north-west and south-west Cameroon are mainly poor subsistence farmers and often live far away from the treatment centre. Guardians have to stay in hospital with the patient, provide meals for their child and pay the hospital bills. In this study free treatment was provided.

Malnutrition (weight for age $<5$ th centile) was present in $50 \%$ of patients with BL in Malawi and in $30 \%$ of patients with BL in Cameroon on admission. ${ }^{3.6}$ Tolerance for chemotherapy and survival

Copyright: (c) 2018. The Authors. Licensee: AOSIS. This work is licensed under the Creative Commons Attribution License. 
rates are influenced by the nutritional status. ${ }^{78,9,10}$ Malnutrition is associated with a higher rate of neutropenia and serious infections, treatment delays, reduced chemotherapy tolerance and a poorer outcome. ${ }^{8}$ The impact of nutritional status on outcomes in children with cancer is an important but neglected area of research. ${ }^{11}$ Prevention of early deaths during the induction phase of treatment is important to improve the overall survival rate.

The majority (85\%) of BL patients have advanced disease and a large abdominal facial tumour burden, and they may eat and drink poorly during the first 3 days of treatment.,

Weight for age $(\mathrm{W} / \mathrm{A})$ is an inaccurate parameter of nutrition in the acute setting. Weight for height $(\mathrm{W} / \mathrm{H})$ is a better parameter of nutrition but may be influenced by factors such as tumour mass, oedema and ascites on admission in children with large tumours. The triceps skinfold (TSF), an indicator of body fat, and the mid-upper arm circumference (MUAC), an indicator of lean body mass, correlate with the golden standard of body composition, the DEXA scan, and are more appropriate objective measurements of nutrition. ${ }^{11,12,13}$ The 5 th centile for TSF in patients aged 4 to 14 years ranges from $5 \mathrm{~mm}$ to $6 \mathrm{~mm}$ in boys and from $6 \mathrm{~mm}$ to $8 \mathrm{~mm}$ in girls. The 5 th centile for MUAC ranges from $126 \mathrm{~mm}$ to $172 \mathrm{~mm}$ in boys and $115 \mathrm{~mm}$ to $174 \mathrm{~mm}$ in girls. ${ }^{14}$

The objective of this descriptive prospective study was to document the anthropometric parameters and co-morbidities in newly diagnosed BL patients, to record morbidity of treatment and to measure the changes in anthropometric parameters during the 28-day induction treatment period in patients who received a standardised treatment protocol and a daily dietary supplement of protein, minerals and vitamins.

\section{Methods}

The study was supervised by a resident paediatrician (MT). Seventy-two consecutive patients were admitted with BL between April 2010 and March 2011 to the Banso, Mbingo and Mutengene Baptist Hospitals in the north-west and southwest regions of Cameroon. Two patients who died of advanced disease within $24 \mathrm{~h}$ of admission were excluded from anthropometric analysis. Standard diagnostic procedures on admission included fine needle aspiration (FNA), an abdominal ultrasound (US) with measurement of the largest intra-abdominal mass, bone marrow aspiration (BM), cerebrospinal fluid (CSF) cytology, a full blood count (FBC), malaria smear, sickle cell test, human immunodeficiency virus (HIV) screening test, urinalysis and microscopy of faeces. The FBC was repeated before every chemotherapy pulse on days 8 and 14 and when fever occurred. The St Jude staging system was used. ${ }^{15}$

All patients were treated with the 2008 Cameroon BL protocol. Induction therapy commenced with $48 \mathrm{~h}$ of parenteral hydration at $3 \mathrm{~L} / \mathrm{M}^{2}$ and oral allopurinol to prevent tumour lysis. A metoclopramide tablet was given $2 \mathrm{~h}$ before and $4 \mathrm{~h}$ after chemotherapy to prevent vomiting. Furosemide was used when needed to achieve a target urinary output of $\geq 3 \mathrm{~mL} / \mathrm{kg} / \mathrm{h}$ during the first $48 \mathrm{~h}$. Chemotherapy was started $24 \mathrm{~h}$ after the onset of hydration and consisted of $40 \mathrm{mg} / \mathrm{kg}$ CPM (oral or intravenous), $12.5 \mathrm{mg}$ intrathecal methotrexate and $12.5 \mathrm{mg}$ intrathecal hydrocortisone on days 1, 8 and 15 . The response to treatment was assessed on Day 28 by clinical assessment and abdominal US prior to starting maintenance chemotherapy. The response to and outcome of treatment will not be discussed in this article.

Every patient received a boiled egg, $200 \mathrm{~mL}$ of World Health Organization (WHO) F-75 formula and a multivitamin tablet as inpatients every day during the first 14 days, and guardians were provided with eggs and multivitamin tablets on discharge to be given daily at home until the follow-up visit on Day 28. Guardians, who have to stay with the patient in the ward, received a daily ration of three cups of dry rice and $\$ 1$ in cash to buy and prepare local food for their child and themselves. Parenteral nutrition was not available. Parents in general strongly object to nasogastric tube feeding, and this was rarely used.

Anthropometric observations included measurement of weight (W) in $\mathrm{kg}$ and height $(\mathrm{H})$, MUAC and TSF in $\mathrm{cm}$, with a standardised scale, Harpenden calliper and a United Nations Children's Fund (UNICEF) measuring tape on days 1 and 28. Nurses were trained to perform these measurements. Two successive measurements were performed and the average value was recorded. Percentiles of W/A, H/A and body mass index (BMI) were determined with WHO Anthroplus software. Weight-for-age percentiles were only determined in children $\leq 10$ years because WHO standards above age 10 years are not available. ${ }^{14,15}$

Coexisting diseases such as malaria and urinary or intestinal parasites were treated. A blood transfusion was considered when the haemoglobin dropped $\leq 7 \mathrm{~g} / \mathrm{dl}$. Neutropenia was defined as a total white blood cell count of $\leq 1.0 \times 10^{9} / \mathrm{L}$. Fever was defined as a single episode of a temperature of $\geq 38.5^{\circ} \mathrm{C}$ or two episodes $\geq 38^{\circ} \mathrm{C}$ within a 24-hour period. A malaria smear was performed immediately, and treatment was given if found positive. If negative, first-line antibiotic treatment with intravenous gentamicin and ampicillin was started immediately irrespective of the white cell count. Blood cultures were not available. A cephalosporin was added if fever persisted $>48 \mathrm{~h}$. Mucositis was treated with oral nystatin and acyclovir. The full cost of treatment, including hospitalisation, investigations, all medicines and transport for follow-up visits, was provided at no cost to the guardians.

The treatment protocol was approved by the Institutional Review Board of the Cameroon Baptist Convention and parents gave informed signed consent.

\section{Results}

The 70 study patients included 42 boys and 28 girls with a mean age of 8 (range 2 to 16) years. Burkitt lymphoma was 
confirmed on FNA in $76 \%$ and in the remainder on BM, CSF, abdominal US and clinical presentation. A marked reduction in tumour volume within $48 \mathrm{~h}$ was accepted as confirmation of the diagnosis in patients with a clinical diagnosis. The St Jude stage distribution was as follows: Stage I $=4(6 \%)$, Stage II $=3(4 \%)$, Stage III = $48(69 \%)$ and Stage IV = $15(21 \%)$. The US volume of the largest single abdominal mass (there usually are multiple masses) was $>400 \mathrm{~mL}$ in $32 \%$ of patients and $<400 \mathrm{~mL}$ in $49 \%$ of patients. The volume of the largest abdominal mass recorded exceeded $3000 \mathrm{~mL}$. Abdominal US indicated ascites in two patients and a small pleural effusion in one patient.

On admission $\mathrm{H} / \mathrm{A}$ was less than the 10th centile in 28 $(42 \%), W / A$ less than the 10th centile in $12(18 \%)$, and BMI less than the 10 th centile in $50 \%$ of patients aged $>10$ years. The TSF was less than the 3rd WHO centile in $46 \%$, and the MUAC less than the 3 rd WHO centile in $16 \%$ of patients. ${ }^{16}$ On Day 28 the TSF had increased by $0.5 \mathrm{~cm}$ in $69 \%$, and the MUAC was now less than or equal to the 3 rd centile in $10 \%$ of patients (Table 1). Weight loss of $\geq 5 \%$ of body weight was recorded in $42 \%$ of patients, and weight gain in $24 \%$ of patients. Fifty per cent of patients, with a largest recorded single abdominal tumour of $<400 \mathrm{~mL}$ in volume, gained weight during induction therapy. Five of seven patients without abdominal masses (St Jude Stages I and II) gained $>1 \mathrm{~kg}$ in weight, and six of these patients had an increase in TSF of $>5 \mathrm{~mm}$. Anthropometric data are summarised in Table 1, and individual patients' changes in TSF and MUAC are illustrated in Figure 1. Weight and height were not recorded in two and four patients, respectively, on admission, and height on Day 28 was not recorded in 11 patients.

Two patients (3\%) died from presumed tumour lysis and renal failure shortly after the first course of chemotherapy. Co-morbidities at diagnosis included malaria, sickle cell trait, intestinal parasites, schistosomiasis and HIV sero-positivity and are listed in Table 2. Three patients developed renal failure after the first dose of chemotherapy, of whom two died within the first week. Neutropenia occurred in $12(18 \%)$, febrile episodes in $42(60 \%)$ and prolonged fever in $17(24 \%)$ of patients. Fourteen patients $(20 \%)$ received a blood transfusion. Other complications observed were diarrhoea and vomiting, mucositis, cough, dental problems, wound infection, chickenpox, skin infection and anal prolapse

TABLE 1: Anthropometric parameters on days 1 and 28.

\begin{tabular}{lcc}
\hline Parameters & Day $\mathbf{1}(\mathbf{\%})$ & Day 28 (\%) \\
\hline Weight for age $<10$ th centile & 18 & - \\
Weight gain $\geq 5 \%$ & - & 21 \\
Weight loss $\geq 5 \%$ & - & 34 \\
Weight unchanged & - & 45 \\
Height for age $<10$ th centile & 42 & - \\
Body mass index $<10$ th centile & 50 & - \\
MUAC $<3$ rd centile & 16 & 10 \\
TSF increase $>0.5 \mathrm{~cm}$ & - & 57 \\
TSF $<3$ rd centile & 47 & - \\
\hline
\end{tabular}

MUAC, mid-upper arm circumference; TSF, triceps skinfold. (which corrected spontaneously) (see Table 3). No other deaths occurred during the 28-day induction treatment period.

The limitations of this study, such as the unavailability of microbiological cultures, an intensive care unit, renal dialysis and parenteral feeding, were a result of the limited resources that were available in this setting at the time. We did not record the type and quantity of food that the parents gave to their children in addition to the daily dietary supplements.

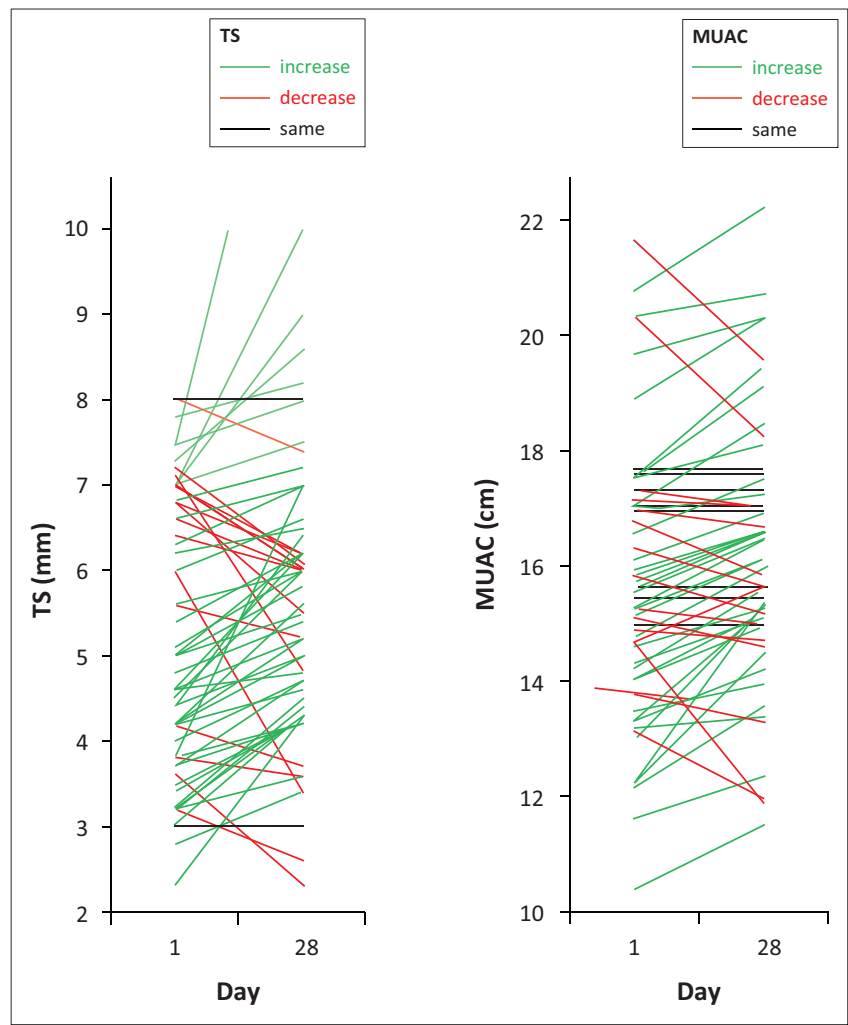

MUAC, mid-upper arm circumference; TSF, triceps skinfold.

FIGURE 1: Changes in triceps skinfold and mid-upper arm circumference.

TABLE 2: Co-morbidities on admission in 70 patients.

\begin{tabular}{lcc}
\hline Co-morbidity & Number & Percentage \\
\hline Malaria & 6 & 9 \\
Sickle cell trait & 5 & 7 \\
Intestinal parasites & 7 & 10 \\
Schistosomiasis & 1 & 1 \\
HIV seropositive & 2 & 2 \\
\hline
\end{tabular}

HIV, human immunodeficiency virus.

TABLE 3: Morbidity during treatment.

\begin{tabular}{lcc}
\hline Event & Number & Percentage \\
\hline Febrile episode & 42 & 60 \\
Fever $>48 \mathrm{~h}$ & 17 & 24 \\
Neutropenia & 12 & 18 \\
Diarrhoea, vomiting, gastroenteritis & 9 & 13 \\
Blood transfusion & 14 & 20 \\
Mucositis & 4 & 6 \\
Cough & 2 & 3 \\
Dental problems & 2 & 3 \\
Varicella & 1 & 1 \\
Skin infection & 1 & 1 \\
Wound infection & 2 & 3 \\
Anal prolapse & 1 & 1 \\
\hline
\end{tabular}




\section{Discussion}

The H/A was less than the 10th centile in $42 \%$ and the W/A less than the 10 th centile in $18 \%$ of patients. This confirms the high prevalence of poor nutrition in patients with BL at diagnosis. However weight reflects the short-term nutritional status at diagnosis and is overestimated in patients who have a large total tumour mass at diagnosis. Weight gain by Day 28 was recorded in $21 \%$ of all the patients but in $71 \%(5 / 7)$ of patients who had no abdominal masses (Stage I and II disease) and in $50 \%$ of children who presented with smaller abdominal tumours. Co-morbidities such as malaria and intestinal parasites, which may adversely affect the nutritional state, were identified and fully treated on admission.

The egg, $200 \mathrm{~mL}$ of F75 and multivitamin tablet, which provided a minimum daily intake of $8 \mathrm{~g}$ of good quality protein and additional vitamins, minerals and trace elements, was supplemented by the food (mainly rice, maize, vegetables and fruit) that the parents prepared.

An increase of the TSF in 57\% of patients, and a reduction in the number of children with a MUAC less than the third centile from $16 \%$ to $10 \%$, indicates that the nutritional support had a positive effect. Fever, febrile episodes and co-morbidities were managed without serious consequences. Laboratory facilities were too limited to accurately quantify renal function.

There were two deaths during induction treatment, constituting a $2.8 \%$ death rate during treatment. If the two patients who died before chemotherapy was commenced are added, the overall death rate was 5.5\% (4/72). No patients abandoned treatment. This death rate is significantly lower $(P=0.009, p<0.5)$ than the $8.5 \%(24 / 129)$ rate recorded in a cohort of patients who had previously been treated with the same chemotherapy protocol at the same hospitals, but for whom the nutritional support provided was limited. ${ }^{3}$ The French-African Paediatric Oncology Group reported a 5.1\% death rate with CPM induction treatment in 178 patients, but their exclusion of 79 patients from the analysis for unspecified reasons does not allow for a fair comparison. ${ }^{17}$ In a recent Malawian study, doxorubicin was added to CPM during induction, and a $12 \%$ death rate was reported during the first 28 days. Ten of the seventy-eight Malawian patients, however, died before treatment was instituted and were not included as early deaths. ${ }^{18}$ All episodes of neutropenia and fever were successfully treated. The two deaths from renal failure may have been prevented if renal dialysis had been available.

We could find no publications on the changes in nutritional status and the effect of dietary support during the first month of treatment in children with endemic BL with which to compare our findings.

Although supplements of protein, vitamins and trace elements were provided, we did not record the type and quantity of food that the parents prepared and gave to their children. We intend to provide an optimal diet to our patients in the future and to educate parents how best to prepare available and affordable local food with the help of trained dieticians.

\section{Conclusion}

We have demonstrated that good enteral nutritional support during induction chemotherapy in a malnourished population of patients with BL resulted in an improvement in nutritional parameters in the majority of patients and was associated with a low morbidity and death rate during induction treatment. Nutritional support should be a standard component of treatment in all children with cancer.

\section{Acknowledgements}

This article was presented in part at the International Society of Paediatric Oncology (SIOP) Africa Continental Conference in Cape Town in 2014. The authors acknowledge the following entities for financial support: Swiss Cancer League, Beryl Thyer Memorial Africa Trust and Stellenbosch University, Burkitt Research Fund.

\section{Competing interests}

The authors declare that they have no financial or personal relationships that may have inappropriately influenced them in writing this article.

\section{Authors' contributions}

P.B.H. was the principal investigator. M.T. was the physician conducting study at the Banso Baptist Hospital. E.L. was responsible for expert advice on nutrition. G.A. was responsible for the data collection and management. F.K. was the physician conducting study at the Mbingo Baptist Hospital. E.K. was the physician conducting study at the Mutengene Baptist Hospital.

\section{References}

1. Steliarova-Foucher E, Colombet M, Ries LAG, et al. International incidence of childhood cancer, 2001 - 2010: A population based study Lancet Oncol. 2017;18(6):719-731. https://doi.org/10.1016/S1470-2045(17)30186-9

2. Lewis N, Young J, Hesseling PB, McCormick P, Wright N. Epidemiology of Burkitt's lymphoma in Northwest Province, Cameroon. Paediatr Int Child Health 2012;33(2):82-85. https://doi.org/10.1179/2046905511Y.0000000016

3. Hesseling PB, Njume E, Kouya F, et al. The Cameroon 2008 Burkitt lymphoma protocol: Improved event - Free survival with treatment adapted to disease stage and the response to induction therapy. Pediatr Hematol Oncol. 2012;29:119-129. https://doi.org/10.1179/2046905511Y.0000000016

4. Patte $C$, Auperin A, Michon J, et al. The Societe Francaise d'oncoligique pediatrique LMB protocol: Highly effective multi-agent therapy tailored to the tumour burden and initial response in 561 unselected children with B - Cell lymphoma and L3 leukaemia. Blood. 2001;97:3370-3379. https://doi.org/10.1182/blood.V97.11.3370

5. Reiter A, Schrappe P, Tiemann M, et al. Improved treatment results in childhood B - Cell lymphoma with tailored intensifications of treatment. A report of the Berlin - Frankfurt Munster group trial NHL BFM 90. Blood. 1999;94:3294-306.

6. Hesseling P, Molyneux E, Kamisa S, Israels T, Broadhead R. Endemic burkitt lymphoma: A 28- day treatment schedule with cyclophosphamide and intrathecal methotrexate. Ann Trop Paediatr. 2009;29:29-34. https://doi.org/10.1182/blood. V97.11.3370

7. Rickard KA, Detamore CM, Coates TD, et al. Effect of nutrition staging on treatment delays and outcome in stage IV neuroblastoma. Cancer. 1983;52(4):587-598. https:// doi.org/10.1002/1097-0142(19830815)52:4\%3C587::AID-CNCR2820520402\% 3E3.0.CO;2-T

8. Israels T, Van de Wetering MD, Hesseling P, Van Geloven N, Caron HN, Molyneux EM. Malnutrition and neutropenia in children treated for Burkitt lymphoma in Malawi. Pediatr Blood Cancer. 2009;53(1):47-49. https://doi.org/10.1002/ pbc.22032 
9. Katona $P$, Katona-Apte J. The interaction between nutrition and infection. Clin Infect Dis. 2008;46(10):1582-1588. https://doi.org/10.1086/587658

10. Scrimswaw NS, Taylor CE, Gordon JE. Interactions of nutrition and infection. Am J Med Sci. 1959;237(3):367-403.

11. Rogers PC, Ladas EJ. The impact of nutritional status on outcomes: A neglected ar of research. Pediatr Blood Cancer. 2011;57:902-903. https://doi.org/10.1086/ 587658

12. Mosby TT, Bar RD, Pencharz PB. Nutrtional assessment of children with cancer J Pediatr Oncol Nurs. 2009;26:186-197. https://doi.org/10.1177/1043454209340326

13. Berkley J, Mwangi I, Griffiths K, et al. Assessment of severe malnutrition amon hospitalized children in rural Kenya: Comparison of weight for height and mid upper arm circumference. JAMA. 2005;294(5):119-129. https://doi.org/10.1001/ jama.294.5.591
14. Frisancho AR. New norms for of upper limb fat and muscle areas for assessment of nutritional status. Am J Clin Nutr. 1981;34:2520-2545. https://doi.org/10.1093/ ajcn/34.11.2540

15. Murphy SB. Classification, staging and end results of treatment of non-Hodgkin lymphomas in childhood: Dissimilarities from lymphomas in adults. Semin Oncol. 1980;7:332-339.

16. World Health Organization. Child growth standards. Hoboken, NJ: Wiley; 2006.

17. Traore F, Coze C, Atteby JJ, et al. Cyclophosphamide monotherapy in children with Burkitt Lymphoma: A study from the French African Pediatric Oncology Group (GFAOP). Pediatr Blood Cancer. 2011;56:70-76. https://doi.org/10.1002/pbc.22746

18. Molyneux E, Schwalbe ED, Chagaluka G, et al. The use of anthracyclines in the treatment of endemic Burkitt lymphoma. Br J Haematol. 2016;177:984-990. https://doi.org/10.1111/bjh.14440 